Background Many injury victims die before reaching a hospital due to inadequate pre-hospital care and transport. Prompt emergency care can save lives and prevent disabilities but the inconsistent availability of formal Emergency Medical Services (EMS) makes it challenging. This is the case even in some high-income developing countries like Oman. For this study we analysed the trauma registry data from two large hospitals of Oman to understand the pre-hospital and hospital based emergency care in a rapidly developing country in the Arab Gulf.

Methods The data was collected from Khoula and Nizwa hospitals between November 2014 and April 2015. All patients admitted through the emergency department with a history of trauma were included. Information about mechanism of injury, mode of transport, time interval between injury and hospital, pre-hospital care and emergency department (ED) disposition was collected.

Results 2,340 patients were received in the ED during the study period. The majority (74\%) were males, with a mean age 27 years. Transport injuries and falls accounted for $70 \%$ of all injuries. The most common mode of transport was private car $(43 \%)$; only $13 \%$ of patients were transported via EMS. Only $30 \%$ of cases were transported to the hospital within an hour of injury; median transport time was 2.53 hours. Only $27 \%$ of patients received some form of pre-hospital care. Twenty-three ED deaths were recorded. Mean ED length of stay was 16.35 hours. In the study population, injury severity score in $85 \%$ of cases was $\leq 9$, mean revised trauma score was 7.6382 and overall mortality ratio was $2.35 \%$.

Conclusion Despite rapidly developing health care services in the urban parts of Oman, EMS utilisation is low. Patients presenting to the hospital are those who have less severe injuries and thus have better chances of survival. A better-organised EMS system may provide a prompt transport and appropriate triage to patients with severe injuries.

\section{RECOVERY AFTER SERIOUS TRAUMA - OUTCOMES, RESOURCE USE AND PATIENT EXPERIENCES (RESTORE) STUDY: 3-YEAR OUTCOMES FOR ADULTS}

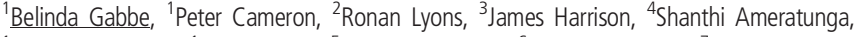
${ }^{1}$ Jennie Ponsford, ${ }^{1}$ Alex Collie, ${ }^{5}$ Mark Fitzgerald, ${ }^{6}$ Rodney Judson, ${ }^{7}$ Andrew Nunn, ${ }^{8}$ Nicola Christie, ${ }^{1}$ Sandy Braaf, ${ }^{9}$ David Attwood, ${ }^{10}$ Helen Jowett, ${ }^{10}$ Warwick Teague. ${ }^{1}$ Monash University, Australia; ${ }^{2}$ Swansea University, United Kingdom; ${ }^{3}$ Flinders University, Australia; ${ }^{4}$ University of Auckland, New Zealand; ${ }^{5}$ The Alfred, Australia; ${ }^{6}$ Royal Melbourne Hospital, Australia; ${ }^{7}$ Victorian Spinal Cord Service, Australia; ${ }^{8}$ University College London, United Kingdom; ${ }^{9}$ Transport Accident Commission, Australia; ${ }^{10}$ Royal Children's Hospital, Australia
\end{abstract}

\subsection{6/injuryprev-2016-042156.307}

Background There is a worldwide shortage of population data about non-fatal injury burden. The aim of this population-based, nested, longitudinal study was to quantify patient outcomes in the first 5 years after injury.

Methods Adult ( $>17$ years), major trauma patients registered by the population-based Victorian State Trauma Registry, injured between July 2011 and June 2012, were followed up by telephone at 6-months and 1,2, 3, 4 and 5 years post-injury to collect function (GOS-E), health status (EQ-5D), return to work, environmental impact (CHIEF-SF) and post-traumatic stress disorder (IES-R) data. Data from the 3-year time point are presented.
Results Of 2,757 patients, 333 died in-hospital and 99 opted-out of follow-up. Of the remaining 2,325 patients, $10 \%$ died before 3 years. The mean (SD) age of surviving patients was 49 (21) years, $73 \%$ were male, $89 \%$ were unintentional cases. Road traffic injury (37\%) and falls (32\%) were common causes. $62 \%$ were working/studying before injury. $84 \%$ were followed up at 3 years. $29 \%$ reported upper good recovery on the GOS-E, and $69 \%$ of those working/studying before injury had resumed work/study. Prevalence of problems on the EQ-5D items varied; 37\% for mobility, $21 \%$ for personal care, $47 \%$ for usual activities, $50 \%$ for pain/discomfort and $41 \%$ for anxiety/depression. Mean CHIEF-SF scores indicated greater problems than the CHIEF norms for the transport $(0.77$ vs 0.48$)$, natural environment (1.26 vs 0.63$)$, physical structure of other surroundings (1.00 vs $0.44)$, home help (0.51 vs 0.35$)$ and health care (0.49 vs 0.34$)$ items. IES-R scores for $43 \%$ showed no symptoms; $21 \%$ had some symptoms and 18\% scored $>33$ which is indicative of a clinical diagnosis of PTSD.

Conclusions At 3-years post-injury, ongoing problems were prevalent. The data provides valuable information about the time course for recovery and key problems faced by patients in their recovery.

\section{EPIDEMIOLOGICAL TRENDS IN THE SWISS REHABILITATION SETTING FOR TRAUMATIC SPINAL CORD INJURY}

1,2 Jonviea Chamberlain, 1,2Martin WG Brinkhof. 'Swiss Paraplegic Research, Nottwil, Switzerland; ${ }^{2}$ University of Lucerne, Switzerland

\subsection{6/injuryprev-2016-042156.308}

Background Dynamics of incident traumatic accidents, a case in point being traumatic spinal cord injury (TSCI), have changed over the past forty years in many high income countries due to changes in demographic structure, lifestyle and policy. Rehabilitation following a TSCI can result in significant improvements and prolongation of life and reduced societal and personal burden. Monitoring contemporary and historical trends can help inform future projections for targeted interventions towards prevention and resource allocation.

Methods TSCI cases admitted for first rehabilitation between 1970 and 2014 were identified in four specialised rehabilitation centres in Switzerland included in the Swiss Spinal Cord Injury (SwiSCI) cohort. Multinomial logistic regression was used to determine relative risk ratios (RRR) of admission to first rehabilitation. Incidence rate ratios (IRRs) were calculated using Poisson regression with reference to the Swiss general population.

Results There were 4,095 incident cases of TSCI. Between 1971-2000 there was an overall 2.1\% increase in patient admission, followed by a $1.2 \%$ decrease in 2000-2014. The relative proportion of the elderly patient population increased from 1990 onwards in comparison with 1970-1974 (RRR range compared to the youngest: 4.2-14.6). Relative to the youngest patients, the oldest patients had a nearly 18 -fold increase in odds for falls in comparison with transport-related accidents. Assessment of IRRs revealed an increase in risk of admission for sports-related accidents TSCIs among the youngest ages; risk of falls among older ages appeared to increase, especially after 2000. 
Conclusions This study provides evidence for increasing admission to first rehabilitation of the elderly for TSCIs, which can in part be attributed to an increase in risk of TSCI with increasing age. Potential lifestyle changes among the youngest, especially for sports and leisure-related TSCIs, as well as the oldest, possibly due to increase in activity, could contribute to the observed increase in risk.

\section{IMPLEMENTATION AND EVALUATION OF AN MHEALTH SYSTEM FOR ACUTE BURN INJURIES IN RESOURCE- CONSTRAINED SETTINGS}

${ }^{1}$ Marie Hasselberg, ${ }^{2}$ Lee Wallis, ${ }^{3}$ Hendry Sawe, 'Lucie Laflamme. 'Karolinska Institutet, Sweden; ${ }^{2}$ Stellenbosch University; ${ }^{3}$ Muhimbile University

\subsection{6/injuryprev-2016-042156.309}

Background Burn injuries are a leading cause of premature death worldwide and are largely attributable to poor living conditions. Timely care is a prerequisite to reduce morbidity and mortality and it can be significantly improved by smartphone-based consultation systems. The overall aim of this project is to assess how an mHealth system for acute burn injury care can impact on the delivery of emergency care for burns victims in terms of clinical management.

Methods The clinical quality includes assessments of diagnostic accuracy and patient management. Bedside diagnosis or image based diagnosis by burns experts will be used as gold standard. Technology Usability Evaluation Model will be used to assess how the users experience the system.

Results A tele-consultation system for acute burn care is currently under implementation in the Western Cape, South Africa. An application (app) is installed on a smartphone located in each of the emergency services of 8 hospitals to transmit visual and textual information between emergency staff at point of care and a network of burns specialists. A stepwise implementation of the system will start in Tanzania in the beginning of 2016. The burn specialists already involved in South Africa will act as experts for Tanzania. The evaluation will build on an evaluation already started in South Africa. The first part of the evaluation will include the hospitals currently using the mHealth system in the Western Cape, South Africa and 2-3 hospitals in Tanzania. The mHealth system and results of the evaluation will be presented at the conference.

Conclusions Low cost and timely alternatives to burn injury control are a pressing need in many low- and middle-income settings and countries. This project is a determinant step in that direction and can lead to the implementation of a viable, inclusive, and environment friendly mHealth system.

\section{COMMON MORTALITY MODELS FAIL TO USE ANATOMIC INJURY INFORMATION OPTIMALLY}

${ }^{1}$ Alan Cook, ${ }^{2}$ Turner Osler, ${ }^{3}$ Laurent Glance, ${ }^{4}$ Fiona Lecky, ${ }^{5}$ Omar Bouamra. ${ }^{1}$ Chandler Regional Medical Centre, USA; ${ }^{2}$ University of Vermont, USA; ${ }^{3}$ University of Rochester, USA; ${ }^{4}$ University of Sheffield, UK; ${ }^{5}$ University of Manchester, UK

\subsection{6/injuryprev-2016-042156.310}

Background Comparison of trauma centres requires accurate injury severity metrics. The Trauma Audit and Research Network (TARN) in the UK, and the Trauma Injury Severity Score (TRISS) provide such risk adjustment. These models incorporate the Injury Severity Score (ISS) as the measure of anatomic injury severity but categorise it differently. The Trauma Mortality
Prediction Model (TMPM) better predicts mortality than the ISS. We compared the anatomic injury components of TARN, and TRISS models to TMPM.

Methods Data from the National Trauma Data Bank for 20022012. Probability of death was estimated for TARN, and TRISS from ISS values according to each model's treatment and compared these to TMPM using measures of discrimination (area under ROC curves), proximity to the true model (Akaike Information Criterion (AIC)), and calibration (Hosmer-Lemeshow statistic (HL)). Confidence intervals (CI) were estimated by bootstrapping. ISS and TMPM values were estimated for the Abbreviated Injury Scale (AIS) and the International Classification of Diseases, $9^{\text {th }}$ Revision (ICD-9) lexicons. Data are shown as medians and 95\% CI.

Results $\mathrm{N}=1,145,959$

\begin{tabular}{lllllll}
\hline AIS & ROC & \multicolumn{3}{l}{ AIC } & HL & \\
\hline TMPM & 0.8716 & $0.8695-0.8734$ & 238130 & $236110-240201$ & 226.5 & $168.5-289.7$ \\
TARN & 0.8374 & $0.8351-0.8396$ & 270017 & $267958-272213$ & 668.5 & $586.0-745.4$ \\
TRISS & 0.8368 & $0.8346-0.8389$ & 275098 & $273058-277265$ & 2335.3 & $2186.9-2516.4$ \\
ICD-9 & ROC & & AIC & & HL & \\
TMPM & 0.8475 & $0.8451-0.8498$ & 211424 & $209531-213377$ & 107.1 & $76.1-142.3$ \\
TRISS & 0.8234 & $0.8211-0.8258$ & 223081 & $221132-225128$ & 1850.0 & $1702.9-1998.7$ \\
TARN & 0.8131 & $0.8106-0.8153$ & 227002 & $225115-228955$ & 1033.1 & $929.0-1152.4$ \\
\hline
\end{tabular}

Conclusions TMPM better predicted mortality than did the anatomic injury component of TRISS, or TARN and this was true whether individual injuries were coded in the AIS lexicon or in the ICD-9. As trauma care evolves, so must models of mortality prediction that best capture injury severity.

\section{Injury Statistics}

\section{Parallel Tue 2.6}

\section{APPLICATION OF SPATIAL EPIDEMIOLOGICAL APPROACHES TO INJURY RESEARCH: A SYSTEMATIC REVIEW}

Himalaya Singh, Lauren V Fortington, Caroline Finch. Federation University Australia

\subsection{6/injuryprev-2016-042156.311}

Background Injuries are a leading cause of death and disability around the world. Previous studies have shown that certain populations are consistently at greater risk of injury. Spatial epidemiological approach provides a way to better understand injury patterns and their associated risk factors at a population level. The aim of this research is to provide a systematic reivew of spatial epidemiological methods applied to injury research.

Methods A search was conducted in three major electronic databases (PubMed, Web of Science and Science Direct), for papers published between 2000-2015 inclusive. Included were papers reporting unintentional injury outcomes, which used geospatial methods for spatial epidemiological analysis. Findings are reported using PRISMA (Preferred Reporting Items for Systematic Reviews and Meta-Analyses) guidelines.

Results From over 10,000 articles, 88 articles met all inclusion criteria. The major categories of injury data that have been reported with geospatial methods were road traffic (52\%), falls $(11 \%)$, burns $(12 \%)$, drowning $(5 \%)$, workplace injuries $(2 \%)$ 